CUAD. CONTAB. / BOCOTÁ, COLOMBIA, 15 (38): 549-573 / JULIO-DICIEMBRE 2014 / 549

\title{
Rankings de los programas de posgrado en contabilidad: análisis de producción docente según publicación en revistas brasileñas (2000-2009)*
}

doi: 10.11144/Javeriana.cc15-38.rppc

\begin{abstract}
Abimael de Jesus Barros Costa
Doctorando del programa de posgrado en transportes del

Departamento de Ingeniería Civil y Ambiental, Univer-

sidad de Brasilia, UnB. Máster en ciencias contables,

Universidad de Brasilia, UnB. Profesor asistente del

Departamento de Ciencias Contables y Actuarias, Univer-

sidad de Brasilia, UnB, Brasil.

Correo electrónico: acosta@unb.br
\end{abstract}

\section{Paulo Roberto Barbosa Lustosa}

Doctor en contabilidad y contraloría, Universidad de São

Paulo, USP. Profesor titular del Departamento de Ciencias

Contables y Actuarias, Universidad de Brasilia, UnB,

Brasil.

Correo electrónico: lustosa@unb.br

\footnotetext{
* Artículo resultado del proyecto de investigación Rankings dos Programas de Pós-graduação Stricto Sensu em Ciências Contábeis: análise da produção docente baseada em periódicos (2000 a 2009). Disertación de maestría, defendida el 27 de enero de 2011. No tuvo institución financiadora.
} 
Resumen El objetivo de la investigación fue describir la producción docente de los posgrados en contabilidad (Programa de Pós-Graduação em Ciências Contábeis, PPGCC), en una ventana de tiempo de 10 años (2000-2009). A partir del análisis de 27 revistas, se creó una base de datos con la metodología de Joshua G. Coyne, Scott L. Summers, Brady Williams y David A. Wood (2009). Para ello, se elaboraron rankings para ayudar a responder la pregunta de investigación. Posteriormente, se realizó un análisis descriptivo de los artículos. El ranking general del PPGCC evidenció que el programa de la USP (Universidade de São Paulo) es el más destacado. Por su parte, el programa de la FURB (Fundação Universidade Regional de Blumenau) resultó sobresaliente en cuanto a la metodología empírica de estudio de caso, mientras que el programa de la UFPE (Universidade Federal de Pernambuco) presenta producción académica relevante en el ámbito de fiscalidad. Los programas se caracterizan por investigación empírica a partir de bases de datos y concentrada en el área de contabilidad financiera. En general, los PPGCC que se destacan son los de la USP, FURB, FUCAPE (Fundação Instituto Capixaba de Pesquisas em Contabilidade, Economia e Finanças), UFMG (Universidade Federal de Minas Gerais) y USP-RP (Universidade de São Paulo-Ribeirão Preto). Entre otros resultados, se constató que tales programas se caracterizan por el elevado número de profesores de tiempo completo y por la predominancia de investigación empírica positivista en finanzas, que constituye el $80 \%$ de las publicaciones. La mayoría de los artículos publicados son de autoría en parejas o cuartetos. Por último, de 2000 a 2009, su desempeño fue en aumento.

Palabras clave Brasil; educación; investigación en contabilidad; programas de posgrado stricto sensu

\section{Rankings of Graduate Accounting Programs: Analysis of Teacher Production According to Publications in Brazilian Journals (2000-2009)}

Abstract The purpose of this research was to describe the teacher production in accounting graduate programs (Programa de Pós-Graduação em Ciências Contábeis, PPGCC) during a ten-year time window (2000-2009). A database was created using the methodology of Joshua G., Coyne, Scott L. Summers, Brady Williams and David A. Wood (2009), through the analysis of 27 journals. Rankings were made for this purpose, to help answer the research question. Afterwards, a descriptive analysis of the articles was made. The general ranking of the PPGCC showed that the program of the USP (Universidade de São Paulo) is the most prominent. In turn, the program of the FURB (Fundação Universidade Regional de Blumenau) is outstanding regarding the case study empiric study methodology, while the program of the UFPE (Universidade Federal de Pernambuco) shows academic production relevant to the field of fiscal studies. A characteristic of the programs is the empiric research based on databases and focused on the financial accounting area. In general terms, the PPGCC that stand out are from the USP, FURB, FUCAPE (Fundação Instituto Capixaba de Pesquisas em Contabilidade, Economia e Finanças) UFMG (Universidade Federal de Minas Gerais) and USPRP (Universidade de São Paulo-Ribeirão Preto). It was confirmed, among other results, that said programs are characterized by the high number of full time teachers and by the predominance of positivist empiric research on finance, which constitutes $80 \%$ of the publications. Most of the published articles are authored by groups of two or four. Lastly, from 2000 to 2009, their performance increased.

Keywords Brazil; education; research on accounting; graduate programs; stricto sensu

\section{Código JEL M 49}


Rankings dos programas de pósgraduação em contabilidade: análise de produção docente segundo publicação em revistas brasileiras (2000-2009)

Resumo O objetivo da pesquisa foi descrever a produção docente dos programas de pós-graduação em contabilidade (Programa de Pós-Graduação em Ciências Contábeis, PPGCC), em uma janela de tempo de 10 anos (2000-2009). A partir da análise de 27 revistas, criou-se base de dados com a metodologia de Joshua G. Coyne, Scott L. Summers, Brady Williams e David A. Wood (2009). Para isso, elaboraram-se rankings para ajudar a responder a pergunta de pesquisa. Posteriormente, realizou-se análise descritivo dos artigos. O ranking geral do PPGCC evidenciou que o programa da USP (Universidade de São Paulo) é o mais destacado. Por sua parte, O programa da FURB (Fundação Universidade Regional de Blumenau) resultou sobressaliente no que diz respeito à metodologia empírica de estudo de caso, enquanto que o programa da UFPE (Universidade Federal de Pernambuco) apresenta produção académica relevante no âmbito de fiscalidade. Os programas caracterizam-se por pesquisa empírica a partir de bases de dados e é concentrada na área de contabilidade financeira. Em geral, os PPGCC que se destacam são os da USP, FURB, FUCAPE (Fundação Instituto Capixaba de Pesquisas em Contabilidade, Economia e Finanças), UFMG (Universidade Federal de Minas Gerais) e USP-RP (Universidade de São Paulo-Ribeirão Preto). Entre outros resultados, constatou-se que tais programas são caracterizados pelo elevado número de professores a tempo completo e pela predominância de pesquisa empírica positivista em finanças, que constitui $80 \%$ das publicações. A maioria dos artigos publicados são de autoria em casais ou quartetos. Por último, de 2000 para 2009, o seu desempenho foi em aumento.

Palavras-chave Brasil; ensino; pesquisa em contabilidade; programas de pós-graduação stricto sensu

\section{Introducción}

La investigación en el área contable creció a partir del año 2000, impulsada por la creación de nuevos programas de posgrado en ciencias contables (Leite-Filho, 2004). Hasta 1988, Brasil contaba solamente con tres publicaciones especializadas en contabilidad. A partir de 1990, ese número aumentó, como afirma Marcelle Colares Oliveira (2002, p. 70). Estudios realizados por Edson Luiz Riccio, Jacira Tudora Carastan y Marici Cristine Gramacho Sakata (1999); Fábio Frezatti (2000); Marcelle Colares Oliveira (2002); Carlos Renato Theóphilo y Sérgio de Iudícibus (2005); Geraldo Alemandro LeiteFilho (2006-2008); José Alonso Borba y Fernando Dal-Ri Murcia (2006); Iracema Marinho (2007); Maurício Vasconcelos Leão Lyrio, José Alonso Borba y Jeane Maria da Costa (2007); Flávia Cruz de Souza, Suliani Rover, Alessandra Vasconcelos Gallon y Sandra Rolim Ensslin (2008); Octávio Ribeiro de Mendonça-Neto, Edson Luiz Riccio y Marici Cristine Gramacho Sakata (2008); y Márcia Maria dos Santos Bortolocci Espejo, Ana Paula Capuano da Cruz, Rosenery Loureiro Lourenço, Tatiane Antonovz y Lauro Brito de Almeida (2009) abordan algunas características de la producción intelectual en el área, pero es la metodología de clasificación el rasgo que distingue tales trabajos.

Con el objetivo de evidenciar las características determinantes de los PPGCC, se estudió la producción científica de los docentes en revistas, entre los años 2000 y 2009. La línea de tiempo se eligió por la intensificación de la literatura científica en contabilidad, en Brasil, después de 2000. Los estudios de los investiga- 
dores Edson Luiz Riccio, Jacira Tudora Carastan y Marici Cristine Gramacho Sakata (1999); Fábio Frezatti (2000); Marcelle Colares Oliveira (2002); y Carlos Renato Theóphilo y Sérgio de Iudícibus (2005) ponen en evidencia este hecho. Para ello se planteó la siguiente pregunta: Con base en las publicaciones seriadas comprendidas entre 2000 y 2009, ¿cuáles son las características de la producción docente de los programas de posgrado en ciencias contables?

El objetivo de la investigación fue describir las características de la producción docente de los PPGCC, a partir de publicaciones seriadas. Esta investigación se justifica porque conocer las características de la producción de una comunidad científica permite analizar los avances y pespectivas del conocimiento acumulado en la contabilidad en Brasil. Para alcanzar este objetivo fue necesario cumplir las siguientes etapas: i) crear una base de datos con información general acerca de los artículos publicados en revistas y clasificarlos de acuerdo con la metodología empleada y el área temática; ii) utilizar la plataforma Lattes ${ }^{1}$ para establecer la vinculación de los docentes en ventanas de tiempo de 3, 6 y 10 años; construir ranking de los PPGCC según la producción de los docentes. La contribución del estudio de la literatura contable se refiere a la descripción, al cambiar el paradigma de la investigación contable de la producción científica brasileña.

1 La Plataforma Lattes es la experiencia del Consejo Nacional Brasileño para el Desarrollo Científico y Tecnológico (CNPq) en la integración de bases de datos de las hojas de vida de los grupos y las instituciones de educación superior de investigación en un único sistema de información de educación.
Este trabajo está estructurado en siete secciones. La primera corresponde a la sección en desarrollo. La segunda es la fundamentación teórica. La metodología será expuesta en la tercera sección, que tratará acerca de los procedimientos del estudio. La recolección y análisis de datos se abordan en la cuarta y quinta sección, respectivamente. En la sexta parte se revelan los resultados del estudio y, por último, en la séptima sección se presentan las consideraciones finales.

\section{Fundamentación teórica}

Las perspectivas teóricas de los filósofos Karl R. Popper (1979), Thomas Kuhn (1979), Imre Lakatos (1979) y Bertrand Russell (1967) son contribuciones relevantes para la filosofía de la ciencia. Aunque los abordajes de estos investigadores fundamentan las discusiones del estudio, no pretendemos explorar totalmente la temática a partir de sus argumentaciones. Popper defiende que la ciencia o el conocimiento científico se desarrollan sobre la base de la falsabilidad, en vista de la cual cualquier hipótesis debe ser considerada verificable (Francelin, 2004).

Para Thomas Kuhn (1979), la ciencia no evoluciona de forma continua, sino en saltos, mediante cambios cíclicos en la comprensión o en los paradigmas. Este estudio se apoya en la teoría de Kuhn, para explicar los cambios en la producción científica brasileña y de los paradigmas de la contabilidad, lo que permite comparaciones entre diferentes intervalos. Imre Lakatos (1979) sostiene la idea de que hay un ambiente competitivo en los programas de 
investigación, lo cual supone la protección de sus teorías o la refutación de todo lo que no representa un avance en el conocimiento. Silveira (1996, p. 5) afirma que las ideas defendidas por Lakatos respaldan que el progreso del conocimiento dependa de la existencia de programas de investigación en competencia. Las heurísticas lakatianas aparecen como reglas metodológicas que guían las instrucciones a seguir. Una heurística negativa indica el camino para evitar la heurística positiva y el camino a seguir.

Esta investigación parte también en la función básica de la ciencia defendida por Bertrand Russell (1967), que es la de brindar a la sociedad la posibilidad de conocer las cosas. La teoría institucional también brinda soporte teórico para el estudio de los cambios institucionales en los PPGCC con el tiempo y de la rivalidad por la cual un investigador tiende a adherirse a la comunidad científica que está progresando. Algunas investigaciones que abordaron la Teoría Institucional desde la óptica del institucionalismo, del contexto organizacional y del campo organizacional contribuyeron a la maduración del tema aplicado a las instituciones de educación superior (Peci, 2005; Machado-da-Silva, Fonseca \& Crubellate, 2005).

$\mathrm{El}$ isomorfismo institucional de los PPGCC puede resultar de la creación de nuevas revistas, la organización de reuniones científicas, los seminarios y las conferencias científicas. A pesar de que las organizaciones estén en un mismo contexto institucional, hay diferencias entre ellas y entre sus modos de actuar y reaccionar. Esas diferencias dependen básicamente de sus características y/o especificidades individuales y conexiones. Así, cada PPGCC pre- senta unas características definidas que, con el tiempo, sufren modificaciones aunque se desarrollen en un mismo contexto institucional (Nogueira, 2007).

A continuación, se presenta la metodología aplicada en este estudio.

\section{Metodología}

Para alcanzar los objetivos del estudio, enunciados anteriormente, se hace necesaria la definición de la metodología de investigación, al igual que el delineamiento claro de los métodos y técnicas adoptados. Para Antônio Carlos Gil (2010), y Eva Maria Lakatos y Marina de Andrade Marconi (2010), los métodos y las técnicas metodológicas proporcionan respuestas a los problemas propuestos.

La investigación propuesta puede clasificarse como descriptiva, en cuanto a sus objetivos y documental, en cuanto a los procedimientos. Se propone el análisis de revistas para la posterior elaboración de los rankings, lo cual reafirma el carácter descriptivo de la investigación, que busca describir, clasificar e interpretar los datos recolectados acerca de la producción docente de los PPGCC.

Además de su aspecto descriptivo, este estudio adopta las características de la investigación documental al fundamentarse en el análisis de artículos científicos y, a partir de allí, extraer información que será utilizada para responder la problemática de la pesquisa. Según Ilse Maria Beuren (2009, p. 90), “organizar información que se encuentra dispersa" es el sentido de la investigación documental, "[...] sirviendo de consulta para estudios futuros". 
La metodología a seguir en este estudio se basa en el modelo de ranking, propuesto en el estudio de Joshua G. Coyne, Scott L. Summers, Brady Williams y David A. Wood (2009), que construyeron, en una ventana temporal de 20 años, el ranking de 40 universidades estadounidenses, según la metodología aplicada y las áreas temáticas de los artículos.

Para la elaboración de los rankings, se optó por tener en cuenta exclusivamente aquella producción científica divulgada en publicaciones seriadas impresas o electrónicas, disponibles online, pues de acuerdo con Marcelle Colares Oliveira (2002, p. 69), "los artículos publicados en revistas representan una parte relevante del flujo de información derivada de la actividad científica de investigación”. Esto se da por hecho teniendo en cuenta el análisis del cuerpo editorial, que es considerado internacionalmente como un indicador de la calidad necesaria para la evaluación competente e imparcial de los trabajos, dado que el cuerpo editorial está conformado por profesionales de idoneidad reconocida en los medios académicos (Oliveira, 2002, p. 39) ["os artigos publicados em periódicos representam uma relevante parte do fluxo de informações originada com a atividade científica de pesquisa"].

El recorte temporal de la investigación se da a partir del año 2000, lo que comprende un período de 10 años, que será dividido en segmentos temporales de 2000 a 2002 (3 años), de 2000 a 2005 (6 años) y, por último, de 2000 a 2009 (10 años). Los artículos analizados son la base para la construcción de los rankings y, a partir de cada revista seleccionada, fueron cla- sificados por medio de categorías según la metodología utilizada y por área temática.

La base de datos fue construida a partir de la información extraída de las publicaciones seriadas científicas relacionadas con el área contable, en la que constan el nombre de la revista, el concepto de la Coordenação de Aperfeiçoamento de Pessoal de Nível Superior, CAPES, el año de publicación, el volumen, el número, el ISSN, el nombre del autor, los nombres de los coautores, el título del artículo, la autoría del artículo, las palabras clave, la metodología utilizada, el área temática, el vínculo académico actual de los autores y coautores, separado en tres ventanas temporales de 3 años, 6 años y 10 años, los títulos de los autores y su ocupación profesional.

Las características de la producción docente representan las variables de la investigación; entre ellas se destacan las estrategias de investigación y las áreas temáticas atribuidas a los artículos publicados en las revistas estudiadas. Las estrategias de investigación utilizadas en este trabajo están basadas en los estudios de Rolf Uwe Fülbier y Thorsten Sellhorn (2009), que indagaron en la producción científica durante los 30 años del Congreso Anual de la Asociación Europea de Contadores, EAA; Carla Cruz y Uirá Ribeiro (2004); y Eva Maria Lakatos y Marina de Andrade Marconi (2010). La definición de las estrategias de investigación adoptadas es un asunto controversial en el medio académico. Cada investigador presenta clasificaciones diferentes. En este trabajo se optó por la siguiente clasificación, en lo referente a estrategias de investigación: teórica, empírica base de datos, empírica levanta- 
miento, empírica estudio de caso y empírica investigación experimental (Anexo A).

Las áreas temáticas que serán abordadas en el estudio son auditoría, contabilidad gerencial, contabilidad financiera, tributación, educación e investigación en contabilidad, entre otras basadas en los estudios de Joshua G. Coyne, Scott L. Summers, Brady Williams y David A. Wood (2009); Rolf Uwe Fülbier y Thorsten Sellhorn (2009, p. 27); Marcelle Colares Oliveira (2002, p. 86) y en las áreas temáticas del 10 Congreso USP de Contabilidad y Contaduría (2010), definidas así para lograr el objetivo propuesto en la investigación (Anexo B).

La medición de las variables se efectuó en tres fases distintas, que serán descritas en detalle a continuación. Como primera fase, se realizó la catalogación de los artículos publicados en las revistas que conforman la población del estudio. En la segunda fase, se procedió al análisis del currículo Lattes de cada docente vinculado a los PPGCC y la posterior comparación entre los artículos catalogados y los datos de los docentes que constituyen la muestra de la investigación. Para Gilberto de Andrade Martins y Carlos Renato Theóphilo (2009, p. 118), "la muestra es un subconjunto de la población". En la tercera fase, cada artículo científico de la muestra fue analizado, en lo que respecta al resumen, las palabras clave, la metodología y los resultados. Después de la recolección, se construyó la base de datos propuesta como herramienta auxiliar en el análisis de los resultados y se elaboraron los rankings.

Los rankings fueron elaborados con base en la muestra de artículos publicados por docentes vinculados a algún PPGCC. En la tabla 1, se presenta la relación de las 27 publicaciones seriadas que fueron empleadas en la construcción de la base de datos y de los rankings, todas ellas clasificadas en la Coordenação de Aperfeiçoamento de Pessoal de Nível Superior del Ministério da Educação, CAPES/MEC, excepto la Revista de Contabilidade y Controladoria, $R C \& C$, a la que fue atribuido un concepto "C" para ajustarse al objeto del estudio.

\begin{tabular}{clccc}
\hline ISSN & \multicolumn{1}{c}{ Nombre de la publicación (institución) } & Concepto & Total & (\%) \\
\hline $1980-4814$ & ABCustos (ABC) & B5 & 41 & 3,50 \\
\hline $1807-7692$ & Brazilian Administration Review, BAR (ANPAD) & A2 & 9 & 0,80 \\
\hline $1807-054 X$ & Base (UNISINOS) & B2 & 50 & 4,30 \\
\hline $1807-734 X$ & $\begin{array}{l}\text { Brazilian Business Review, BBR (edición en portugués, online - } \\
\text { FUCAPE) }\end{array}$ & B2 & 60 & 5,10 \\
\hline $0103-734 X$ & Contabilidade Vista \& Revista (UFMG) & B3 & 127 & 10,80 \\
\hline $1984-3925$ & Contabilidade, Gestão e Governança (UnB) & B4 & 92 & 7,80 \\
\hline $1678-2089$ & Contextus, Revista Contemporânea de Economia e Gestão (UFC) & B4 & 21 & 1,80 \\
\hline $1808-2882$ & Custos e @gronegócio Online (UFRPE) & B4 & 1 & 0,10 \\
\hline $1740-8008$ & $\begin{array}{l}\text { International Journal of Accounting, Auditing and Performance } \\
\text { Evaluation }\end{array}$ & A2 & 2 & 0,20 \\
\hline $1443-9905$ & Journal of Applied Management Accounting Research, JAMAR & C & 48 & 4,10 \\
\hline
\end{tabular}




\begin{tabular}{|c|c|c|c|c|}
\hline ISSN & Nombre de la publicación (institución) & Concepto & Total & (\%) \\
\hline $1981-5700$ & $\begin{array}{l}\text { Revista de Administração Contemporânea Eletrônica, RAC } \\
\text { (ANPAD) }\end{array}$ & B1 & 2 & 0,20 \\
\hline $1415-6555$ & Revista de Administração Contemporânea, RAC (impresa, ANPAD) & B1 & 22 & 1,90 \\
\hline 0034-7590 & Revista de Administração de Empresas, RAE (impresa, FGV) & B1 & 8 & 0,70 \\
\hline $1676-5648$ & $\begin{array}{l}\text { Revista de Administração de Empresas, RAE Eletrônica (online, } \\
\text { FGV) }\end{array}$ & B1 & 9 & 0,80 \\
\hline $1981-8610$ & Revista de Educação e Pesquisa em Contabilidade, REPeC (CFC) & B3 & 4 & 0,30 \\
\hline $1983-8611$ & Advances in Scientific and Applied Accounting (AnpCONT) & $\mathrm{C}$ & 12 & 1,00 \\
\hline $1679-0731$ & Revista Brasileira de Finanças, RBFin (FGV) & B1 & 75 & 6,40 \\
\hline $1519-7077$ & Revista Contabilidade \& Finanças (impresa, FEA-USP) & B1 & 195 & 16,60 \\
\hline 1808-057X & Revista Contabilidade \& Finanças (online, FEA-USP) & B1 & 23 & 2,00 \\
\hline $1807-1821$ & Revista Contemporânea de Contabilidade, RCC (UFSC) & B3 & 49 & 4,20 \\
\hline $1984-6142$ & Revista de Administração (FEA-USP) & B2 & 64 & 5,40 \\
\hline 1984-3704 & Revista de Contabilidade da UFBA, RC (UFBA) & $\mathrm{C}$ & 26 & 2,20 \\
\hline $1516-215 X$ & $\begin{array}{l}\text { Revista de Contabilidade do Mestrado em Ciências Contábeis } \\
\text { (impresa, UERJ) }\end{array}$ & B4 & 34 & 2,90 \\
\hline $1984-6266$ & Revista de Contabilidade e Controladoria, RC\&C (UFPR) & $\mathrm{C}$ & 46 & 3,90 \\
\hline $1982-6486$ & Revista de Contabilidade e Organização, RCO (USP) & B3 & 37 & 3,10 \\
\hline $1809-3337$ & Revista Universo Contábil (FURB) & B3 & 72 & 6,10 \\
\hline $1982-3967$ & Revista de Informação Contábil, RIC (UFPE) & B4 & 46 & 3,90 \\
\hline Total de artí & ículos (muestra) & & 1.175 & 100,00 \\
\hline
\end{tabular}

Tabla 1. Cuantitativo de revistas seriadas y artículos analizados Fuente: elaboración propia, con base en CAPES y WebQualis, 2010

Cada artículo publicado en las revistas descritas en la tabla 1 , que en su conjunto constituye la muestra de la investigación, con base en la producción de cualquier docente vinculado al PPGCC, obtendrá una puntuación de producción docente de acuerdo con la tabla 2. Los puntos fueron atribuidos en una escala de 1 a 10, para que los objetivos propuestos en este trabajo fueran alcanzados. Esta escala está relacionada con la puntuación de las revistas clasificadas por la CAPES. Cada PPGCC obtuvo un puntaje total y el resultado final es revelado en orden lineal, de acuerdo con la sumatoria de los puntos obtenidos.
Con base en el estudio de Joshua G. Coyne, Scott L. Summers, Brady Williams y David A. Wood (2009), cada docente puntúa para el PPGCC al que se encuentra vinculado en la ventana temporal, de acuerdo con el análisis del currículo Lattes y los años de corte 2002, 2005 y 2009. La metodología adoptada en el estudio de Coyne, Summers, Williams y Wood (2009) es clara en que si el docente cambia de vinculación entre las fechas de corte mencionadas, lleva consigo toda su producción intelectual. Este estudio adoptó ese criterio. 


\begin{tabular}{lcc}
\hline Tipo de publicación & Nivel & Puntos \\
\hline \multirow{4}{*}{ Artículos en revistas } & A1 & 10 \\
\cline { 2 - 3 } & A2 & 8 \\
\hline & B1 & 6 \\
\cline { 2 - 3 } & B2 & 5 \\
\hline & B3 & 4 \\
\hline & B4 & 3 \\
\hline & B5 & 2 \\
\hline & C & 1 \\
\hline
\end{tabular}

Tabla 2. Puntaje atribuido a la producción docente en revistas

Fuente: elaboración propia

La clasificación final evidencia el escalafón en tres ventanas de tiempo: la primera de tres años (2000-2002), la segunda de 6 años (2000-2005) y la última de 10 años (20002009). Los rankings se muestran a partir de la ventana de 10 años, de tal modo que si algún PPGCC deja de existir para las fechas contempladas en las ventanas de 6 o 3 años, la puntuación para aquellos docentes que en esos períodos estuvieran vinculados a una institución de educación superior con programa establecido será considerada en la ventana de 10 años. La información será construida de la siguiente forma:

1. Ranking general de publicaciones en revistas por programa de posgrado y número de docentes vinculados, identificado por el símbolo \#.

2. Ranking de los programas de posgrado por metodología empleada.

3. Ranking de los programas de posgrado por área temática.

4. Ranking general de los programas de posgrado por metodología y temática.
5. Análisis descriptivo, distribución de frecuencia, del porcentaje de artículos publicados en las revistas por metodología aplicada.

6. Análisis descriptivo, distribución de frecuencia, del porcentaje de artículos publicados en las revistas por área temática.

7. Análisis descriptivo, distribución de frecuencia, del porcentaje de artículos publicados por metodología aplicada y área temática.

En caso de que un artículo pueda ser clasificado en más de una categoría, será considerado en todas ellas para el análisis del ranking. Los criterios de desempate serán los siguientes:

1. El mayor número total de puntos por docente.

2. Si persiste el empate, la precedencia se establece por orden alfabético.

\section{Recolección de datos}

Este estudio analiza 27 publicaciones seriadas, disponibles online y publicadas en portugués o inglés. La población de artículos científicos del estudio pertenece a las 27 publicaciones seriadas impresas y electrónicas disponibles online. La muestra empleada en esta investigación es la selección de los artículos publicados en aquellas revistas por docentes vinculados a cualquiera de los 18 PPGCC.

La catalogación fue posible gracias a las páginas web de cada revista estudiada, teniendo en cuenta artículos científicos publicados entre los años 2000 y 2009, con el propósito de construir la base de datos. Esta última tiene cuatro 
(4) niveles: (i) datos de la revista, (ii) datos del artículo científico, (iii) datos de la metodología y área temática y (iv) datos del currículo Lattes del docente.

La construcción de la base de datos se dividió en tres (3) fases: (1) catalogación de las revistas; (2) análisis del currículo Lattes de los docentes vinculados a los programas de posgrado en ciencias contables y (3) análisis y clasificación de los artículos científicos publicados por docentes vinculados al PPGCC.

\section{Análisis de los datos}

Las respuestas a la problemática —objeto de la investigación-se obtuvieron a partir del análisis de las características de la muestra. Esta representa, según los parámetros establecidos en el estudio, el total de la producción científica de los docentes vinculados al PPGCC. El objetivo general de la investigación en curso fue describir las características de la muestra, tanto cualitativas como cuantitativas.

Los datos del estudio, en cuanto a autores y coautores, indican que el 34\% de los trabajos presenta autoría en dúos, mientras que el 36\% cuenta con la participación de tres autores. Los trabajos realizados por cuatro autores constituyen el 19\%, y aquellos elaborados de forma individual representan el 5\%, al igual que los quintetos; por último, los sextetos representan el 1\% del total.

En la tabla 3 se reporta la producción docente por autoría de los PPGCC. Se observa que la mayoría de los programas produce artículos con coautores, mayoritariamente dúos o tríos.

Con base en el gráfico 1 se puede afirmar que el 44\% de la población de artículos cata- logados en la primera fase, unos 2.700 artículos, se concentra en las siguientes publicaciones seriadas: Revista de Administração de Empresas, RAE (impresa); Revista Contabilidade \& Finanças (impresa); Revista de Administração Contemporânea, RAC (impresa); Revista de Administração Contemporânea, RAC Eletrônica; Revista Brasileira de Finanças, RBFin; Revista Contabilidade \& Finanças (online); y Revista de Administração de Empresas, RAE Eletrônica (online). Desde ese punto de vista, la revista ABCustos (São Leopoldo), que contiene el 2\% de la población de artículos catalogados, no supera la marca de 150 artículos. Este hallazgo evidencia que otras publicaciones no están siendo consideradas, pues aquellas catalogadas como A2 representan solamente el 3\% del total de artículos publicados.

El número total de docentes vinculados a los PPGCC fue de 237. Esta cifra se obtuvo al analizar los informes de evaluación de los PPGCC, que están disponibles en el sitio web de CAPES (http://www.capes.gov.br/). Estos forman parte del sistema de evaluación de CAPES. El informe presenta los indicadores de cuerpo docente - actuación de cada PPGCC para el año base de 2009.

Una vez en posesión de los datos de los docentes, se dio inicio a la segunda fase, el análisis del currículo Lattes y la catalogación de los datos para la construcción de la base de datos. Estos datos permiten establecer el vínculo académico del docente en 2002, 2005 y 2009. El título más reciente, el programa en que lo obtuvo, el área académica y la ocupación profesional son los datos de investigación necesarios para la elaboración de la base de datos. 


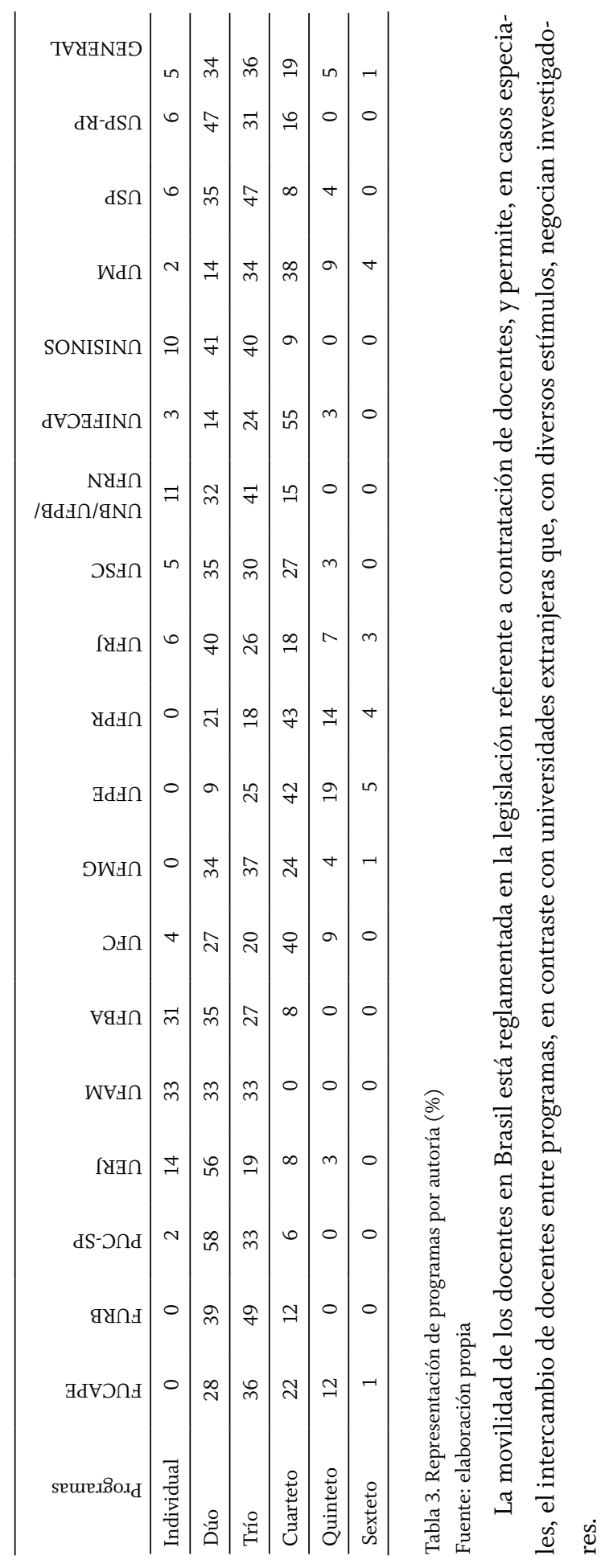




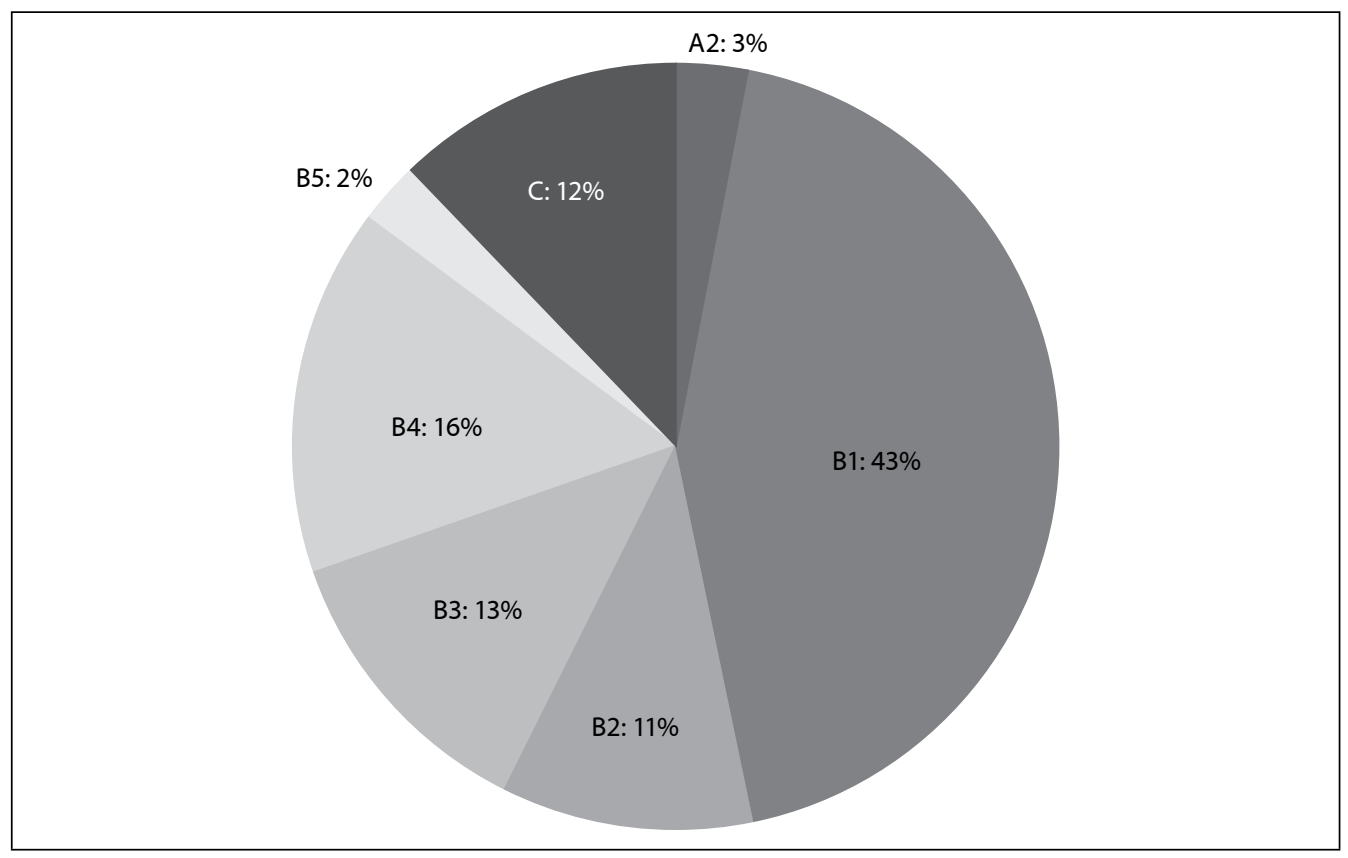

Gráfico 1. Distribución de los artículos publicados por revista Fuente: elaboración propia

Nota: A1, A2, B1, B2, B3, B4, B5 y C representan las clasificaciones de CAPES para publicaciones seriadas

En la mayoría de casos, ese intercambio se da entre docentes de instituciones de educación superior privadas para instituciones federales, estatales o municipales. Es decir, la permuta citada se puede realizar por medio de concurso público con exámenes y títulos.

En este estudio se considera como capacitación continuada la de otros títulos diferentes al doctorado, como posdoctorado o livre-docência (habilitación). Del total de 237 docentes, 129 (55\%) son doctores y 31 (13\%) son doctoras, 41 hombres (17\%) tienen posdoctorado y 9 mujeres (4\%) tienen posdoctorado $y$, por último, 27 (11\%) ostentan livre-docência. Los datos de investigación indican que menos de la mitad de los docentes analizados, el 45\% de la muestra, has- ta el año 2009, habían actualizado el currículo Lattes con información referente a alguna capacitación continuada. De este modo, se entiende que hay limitaciones en la generalización de este tipo de información puesto que, por ejemplo, un docente pudo haber concluido los cursos citados pero sin haber actualizado su currículo Lattes en la fecha de recolección de datos.

Las áreas de formación de la muestra, según los hallazgos del estudio, permiten afirmar que hay una concentración de formación en algunas áreas del conocimiento como contabilidad y contraloría, administración, economía, ingeniería de producción y educación.

Enseguida se presentarán los resultados de la investigación, al igual que su relación con el referente teórico de la pesquisa. 


\section{Resultados}

Una vez concluidas las fases precedentes, catalogación de las revistas seleccionadas y análisis del currículo Lattes de los docentes vinculados a los PPGCC, se seleccionó una muestra de 1.175 artículos publicados por docentes vinculados a los PPGCC.

La producción docente de los 18 PPGCC en el lapso comprendido entre 2000 y 2009 puede ser descrita según 14 características principales, que son abordadas directa o indirectamente en los estudios realizados por José Alonso Borba y Fernando Dal-Ri Murcia (2006); Márcia Maria dos Santos Bortolocci Espejo, Ana Paula Capuano da Cruz, Rosenery Loureiro Lourenço, Tatiane Antonovz y Lauro Brito de Almeida (2009); Fábio Frezatti (2000); Flávia Cruz de Souza, Suliani Rover, Alessandra Vasconcelos Gallon y Sandra Rolim Ensslin (2008); Geraldo Alemandro Leite-Filho (2006), Maurício Vasconcelos Leão Lyrio, José Alonso Borba y Jeane Maria da Costa (2007); Iracema Marinho (2007); Mendonça-Neto, Riccio y Sakata (2008); Marcelle Colares Oliveira (2002); Edson Luiz Riccio, Jacira Tudora Carastan y Marici Cristine Gramacho Sakata (1999); Carlos Renato Theóphilo y Sérgio de Iudícibus (2005); y Silvana Anita Walter, Ana Paula Capuano da Cruz, Márcia Maria dos Santos Bortolocci Espejo y Flavia Pozzera Gassner (2009).

La población de los artículos de investigación científica tiene 27 revistas impresas y electrónicas disponibles en línea. La muestra de esta investigación se basa en la separación de los artículos que fueron publicados por profesores conectados a cualquiera de los PPGCC Brasil, en revistas seleccionadas. La muestra de 1.175 artículos analizados fue seleccionada por el contraste entre la totalidad de los artículos catalogados contenidos en las 27 revistas contempladas y los 237 docentes vinculados a los 18 PPGCC. El análisis de estos artículos completó los parámetros establecidos en la base de datos.

Los datos de la encuesta indican que el área principal de formación de profesores era de contabilidad y control con 101 profesores. En esta concentración hay 73 doctores, 16 profesores asociados y 12 investigadores con posdoctorado. También hay entrenamiento en otras materias como administración de empresas, economía e ingeniería industrial.

Las características de la producción docente de los 18 PPGCC, según el análisis de la muestra de 1.175 artículos publicados en las revistas, son las siguientes: (i) tipo de revista; (ii) concepto CAPES asociado a cada revista; (iii) año de publicación de los artículos; (iv) autores y coautores; (v) título del artículo; (vi) autoría de los artículos; (vii) palabras clave de los artículos; (viii) metodología aplicada; (ix) área temática; (x) vínculo académico; (xi) titulación actual de los autores y coautores; (xii) programa de titulación; (xiii) área de formación; y (xiv) ocupación profesional. Estas variables integran la base de datos.

En la figura 1 aparece la distribución porcentual, por región, de la producción docente de los PPGCC, según la muestra de artículos analizada. La región sudeste exhibe el mayor porcentaje -64,03\%, seguida por la región sur con un porcentaje de 18,84\%; posteriormente aparecen en la secuencia la Región Nordeste con 11,51\%, la Región Centro-Oeste con 5,39 \% y, por último, la Región Norte con el menor porcentaje-0,24\%. 


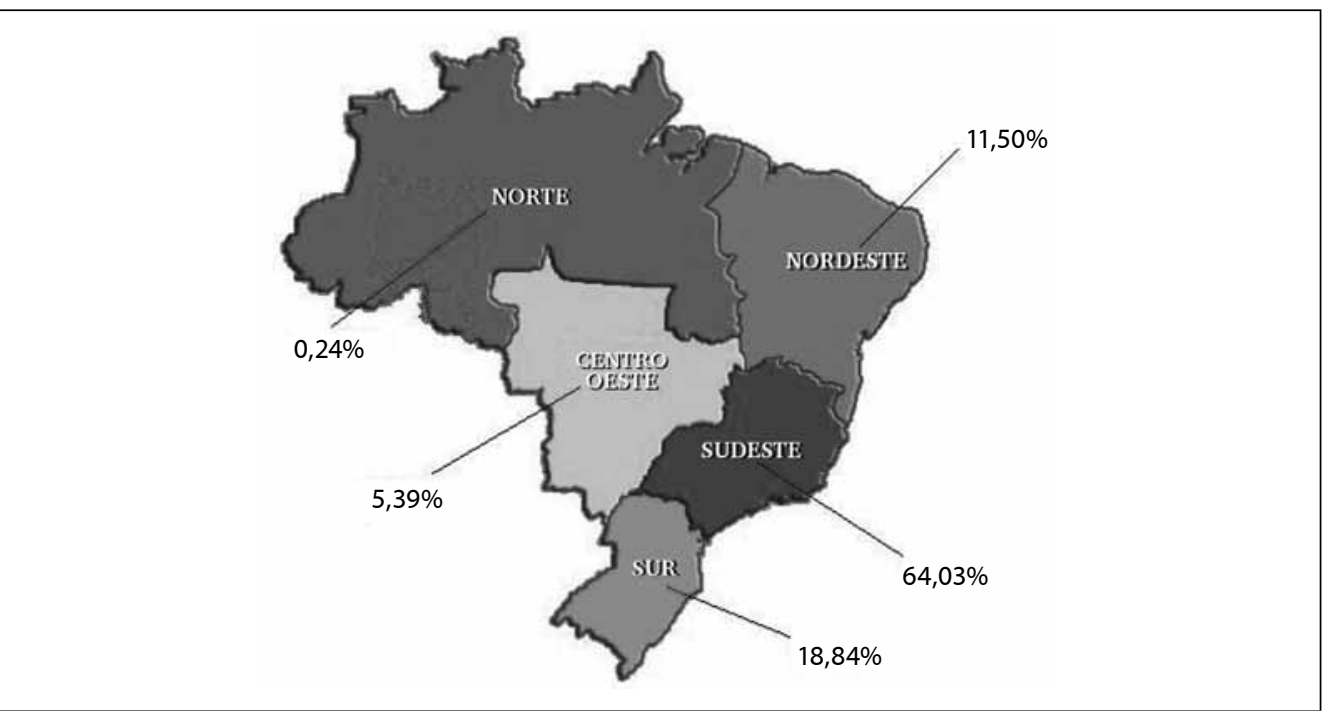

Figura 1. Distribución geográfica de la producción docente de los PPGCC Fuente: elaboración propia

El total de puntos para cada PPGCC en los rankings correspondientes a cada ventana temporal se obtuvo de la siguiente manera: (i) para la ventana temporal de 10 años (2000-2009) se consideraron todos los artículos del período mencionado, es decir, el total de puntos es acumulativo; (ii) para la ventana temporal de 6 años (2000-2005), se tuvieron en cuenta únicamente aquellos artículos publicados en el período indicado; iii) para la ventana de 3 años (2002), solamente fueron considerados aquellos artículos publicados en ese lapso; y (iv) además de los recortes temporales recién citados, se tuvo en cuenta la vinculación de la época, es decir, el intervalo en que el docente estuvo vinculado a algún PPGCC en particular.

El resultado general del ranking de los PPGCC demuestra que el programa de la USP es el más destacado en el período estudiado (20002009). Este programa se mantuvo en primer lugar en todas las ventanas temporales (2002, 2005 y 2009). Los estudios de Ricardo Lopes Cardoso, Octávio Ribeiro de Mendonça-Neto, Edson Luiz Riccio y Marici Cristine Gramacho Sakata (2005); y Octávio Ribeiro de Mendonça-Neto, Edson Luiz Riccio y Marici Cristine Gramacho Sakata (2008) concuerdan con los hallazgos de esta investigación, pues revelan que la FEA-USP fue la institución de educación superior que más contribuyó a la producción científica en contabilidad durante el lapso comprendido entre 1990 y 2005. Los hallazgos incluso señalan que la mayor parte de la producción intelectual de los PPGCC está concentrada en la USP.

El aspecto predominante a la nota sobre la dedicación exclusiva a la enseñanza es la calidad de la educación, al tiempo que reconoce la importancia de una reflexión más larga en esta dimensión. Los profesores relacionados con PPGCC, en su mayoría con dedicación exclu- 
siva, representan el 98\% de la muestra. Algunos PPGCC en Brasil tienen la participación de profesores invitados de Brasil y de otros países. Los programas de la FURB y de la FUCAPE, en el lapso de 10 años, obtuvieron el segundo y tercer lugar en el ranking, respectivamente. Los programas de la UFBA (Universidade Federal da Bahia), UFPR (Universidade Federal do Paraná) y UFAM (Universidad Federal do Amazonas) ocupan las últimas posiciones en los intervalos de diez años y seis años (2005-2009). Estos programas podrían utilizar los resultados de esta investigación para diseñar un plan estratégico para los próximos años.

En general, los datos apuntan hacia la existencia de una concentración de autoría enmarcada en los cinco últimos años de la investigación, de 2005 a 2009, al igual que una concentración en pocos PPGCC. Este resultado es compatible con el estudio de Geraldo Alemandro Leite-Filho (2006), que observa una concentración de la producción científica en la USP, UnB/UFRN/UFPB, UFRJ, UFSC (Universidade Federal de São Carlos), UFPE (Universidade Federal de Pernambuco) y FUCAPE. Esta observación concuerda con el ranking general reportado en la tabla 4.

El resultado evidenciado en el ranking general también concuerda con el resultado de la evaluación trienal (2010) de la CAPES. Los primeros puestos en el ranking general de programas fueron también evaluados por la CAPES. Esto es válido para los programas de posgrado de la USP, FURB, FUCAPE, UFMG (Universidade Federal de Minas Gerais) y USP-RP (Universidade de São Paulo-Riberão Preto) que obtuvieron notas de 6, 4, 4, 4 y 4 otorgadas por la CAPES en $2010^{2}$. Estos cinco PPGCC son responsables por más del 50\% de la producción docente analizada. El resultado del ranking general de los PPGCC se reporta en la tabla 4.

El ranking general de los PPGCC se puede visualizar, de acuerdo con el estudio de Flávia Cruz de Souza, Suliani Rover, Alessandra Vasconcelos Gallon y Sandra Rolim Ensslin (2008), al representar los PPGCC por zonas: central, intermedia y periférica, como se muestra en la figura 2.

Otro resultado que corrobora los hallazgos del estudio es el escalafón de las 400 mejores universidades del mundo divulgado en 2010 por Times Higher Education, THE, que señala a la USP como la universidad brasileña mejor posicionada (puesto 232). La USP fue catalogada como la mejor universidad de América Latina. Entre sus características relevantes, la USP está conformada por docentes de tiempo completo y los docentes tienen título de doctorado como mínimo (Sem Surpresa, 2010).

A manera de síntesis, la tabla 5 evidencia que la USP se destacó entre los demás PPGCC, a pesar de que cuando el análisis se centra en las metodologías empíricas estudio de caso y empírica investigación experimental, las universidades que se destacan son la FURB y la UFSC, respectivamente. De cierto modo, los PPGCC que más se destacaron son aquellos ubicados en áreas geográficas que concentran más del 80\% de la producción docente analizada.

\footnotetext{
2 Nota del traductor: el sistema de calificación de la evaluación trienal de programas de posgrado de las universidades brasileñas realizada por CAPES consiste en una escala de enteros de 1 a 7 , con una nota mínima aprobatoria igual a 3 y nota máxima 7 para programas de calidad y reconocimiento internacional.
} 


\begin{tabular}{|c|c|c|c|c|c|c|c|c|c|c|c|c|c|c|}
\hline \multirow{2}{*}{ Estado } & \multirow{2}{*}{$\begin{array}{l}\text { Estatus } \\
\text { jurídico }\end{array}$} & \multicolumn{3}{|c|}{ CAPES } & \multirow{2}{*}{ Programas } & \multicolumn{3}{|c|}{3 años } & \multicolumn{3}{|c|}{6 años } & \multicolumn{3}{|c|}{10 años } \\
\hline & & $M$ & $\mathrm{D}$ & $\mathrm{F}$ & & TP & $\#$ & $\mathrm{R}$ & TP & $\#$ & $\mathrm{R}$ & TP & $\#$ & $\mathrm{R}$ \\
\hline SP & $\begin{array}{l}\text { Del estado de } \\
\text { São Paulo }\end{array}$ & 6 & 6 & - & USP & 76 & 9 & 1 & 427 & 20 & 1 & 1246 & 21 & 1 \\
\hline $\mathrm{SC}$ & Municipal & 4 & 4 & - & FURB & - & - & - & 102 & 4 & 4 & 395 & 11 & 2 \\
\hline ES & Privada & 4 & 4 & 5 & FUCAPE & 6 & 1 & 10 & 56 & 5 & 10 & 389 & 12 & 3 \\
\hline MG & Federal & 4 & - & - & UFMG & 16 & 2 & 5 & 106 & 4 & 3 & 345 & 9 & 4 \\
\hline SP & $\begin{array}{l}\text { Del estado de } \\
\text { São Paulo }\end{array}$ & 4 & - & - & USP/RP & 20 & 2 & 4 & 70 & 4 & 7 & 326 & 12 & 5 \\
\hline $\mathrm{DF}$ & Federal & 4 & 4 & - & UNB/UFPB/UFRN & 10 & 3 & 8 & 80 & 8 & 5 & 295 & 15 & 6 \\
\hline $\mathrm{PE}$ & Federal & 3 & - & - & UFPE & - & - & - & 56 & 6 & 11 & 275 & 9 & 7 \\
\hline $\mathrm{RJ}$ & Federal & 4 & - & - & UFRJ & 12 & 2 & 6 & 62 & 5 & 8 & 269 & 10 & 8 \\
\hline RS & Privada & 4 & - & - & UNISINOS & 8 & 2 & 9 & 54 & 6 & 12 & 259 & 10 & 9 \\
\hline SP & Privada & - & - & 4 & UPM & 12 & 2 & 7 & 72 & 7 & 6 & 253 & 8 & 10 \\
\hline $\mathrm{SC}$ & Federal & 4 & - & - & UFSC & 40 & 3 & 3 & 39 & 5 & 13 & 240 & 12 & 11 \\
\hline SP & Privada & 3 & - & - & $\mathrm{PUC/SP}$ & 42 & 3 & 2 & 113 & 6 & 2 & 236 & 8 & 12 \\
\hline $\mathrm{CE}$ & Federal & 3 & - & - & UFC & - & - & - & 30 & 7 & 14 & 211 & 12 & 13 \\
\hline SP & Privada & 4 & - & - & UNIFECAP & - & - & - & 15 & 3 & 16 & 134 & 7 & 14 \\
\hline RJ & $\begin{array}{l}\text { Del estado de } \\
\text { Rio de Janeiro }\end{array}$ & 3 & - & - & UERJ & 6 & 2 & 11 & 60 & 8 & 9 & 126 & 10 & 15 \\
\hline $\mathrm{BA}$ & Federal & 3 & - & - & UFBA & - & - & - & 27 & 3 & 15 & 113 & 5 & 16 \\
\hline $\mathrm{PR}$ & Federal & 3 & - & - & UFPR & - & - & - & 6 & 1 & 17 & 93 & 8 & 17 \\
\hline $\mathrm{AM}$ & Federal & - & - & 3 & UFAM & - & - & - & 4 & 1 & 18 & 12 & 2 & 18 \\
\hline
\end{tabular}

Tabla 4. Ranking general de los programas de posgrado Fuente: elaboración propia Nota: M: maestría. D: doctorado. F: maestría profesional. TP: total de puntos. \#: número de docentes. R: ranking

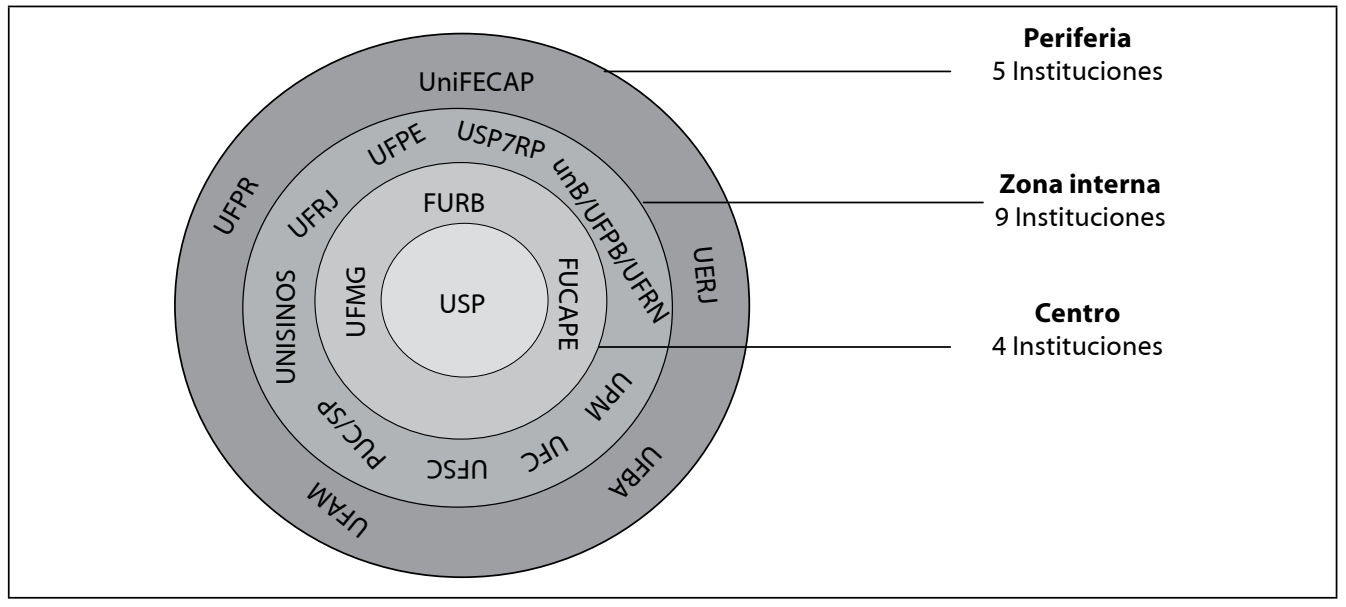

Figura 2. Ranking general de los PPGCC por zona central, intermedia y periférica Fuente: adaptado de Flávia Cruz de Souza, Suliani Rover, Alessandra Vasconcelos Gallon y Sandra Rolim Ensslin (2008) 
RANKING DE LOS PROGRAMAS DE POSGRADO EN CONTABILIDAD EN BRASIL / A. COSTA, P. LUSTOSA / 565

\begin{tabular}{|c|c|c|c|c|c|}
\hline \multirow{3}{*}{ Programas } & \multicolumn{5}{|c|}{10 años } \\
\hline & EBD & $\mathrm{T}$ & EEC & EPE & EL \\
\hline & $\mathrm{R}$ & $\mathrm{R}$ & $\mathrm{R}$ & $\mathrm{R}$ & $\mathbf{R}$ \\
\hline USP & 1 & 1 & 9 & 3 & 2 \\
\hline FUCAPE & 2 & 9 & 13 & 8 & 11 \\
\hline UFMG & 3 & 5 & 3 & 12 & 15 \\
\hline UNISINOS & 4 & 16 & 4 & 10 & 16 \\
\hline USP/RP & 5 & 3 & 7 & 6 & 10 \\
\hline \multicolumn{6}{|l|}{ UNB/UFPB/ } \\
\hline UFRN & 6 & 7 & 11 & 9 & 5 \\
\hline UFPE & 7 & 12 & 6 & 5 & 3 \\
\hline PUC/SP & 8 & 4 & 14 & 14 & 9 \\
\hline UPM & 9 & 8 & 17 & 2 & 7 \\
\hline UFRJ & 10 & 10 & 2 & - & 8 \\
\hline FURB & 11 & 2 & 1 & 4 & 1 \\
\hline UFC & 12 & 11 & 5 & - & 17 \\
\hline UFSC & 13 & 6 & 8 & 1 & 14 \\
\hline UFBA & 14 & 15 & 18 & 7 & 12 \\
\hline UFPR & 15 & 17 & 12 & 13 & 13 \\
\hline UNIFECAP & 16 & 14 & 15 & 11 & 4 \\
\hline UERJ & 17 & 13 & 10 & - & 6 \\
\hline UFAM & 18 & - & 16 & - & - \\
\hline
\end{tabular}

\begin{tabular}{lcccccc}
\hline & \multicolumn{6}{c}{10 años } \\
\cline { 2 - 7 } Programas & CF & OUT & CG & EPC & AUD & TB \\
\cline { 2 - 7 } & $\mathbf{R}$ & $\mathbf{R}$ & $\mathbf{R}$ & $\mathbf{R}$ & $\mathbf{R}$ & $\mathbf{R}$ \\
\hline USP & 1 & 1 & 1 & 1 & 2 & 2 \\
\hline FUCAPE & 2 & 3 & 12 & 15 & - & - \\
\hline USP/RP & 3 & 2 & 11 & 9 & - & - \\
\hline UFMG & 4 & 8 & 3 & 16 & 6 & 4 \\
\hline PUC/SP & 5 & 15 & 9 & 13 & 3 & 10 \\
\hline UFRJ & 6 & 12 & 6 & 14 & - & 7 \\
\hline UNB/UFPB/ & 7 & 14 & 7 & 6 & 7 & 3 \\
\hline UFRN & 8 & 7 & 2 & 3 & 1 & - \\
\hline FURB & 9 & 11 & 10 & - & 8 & - \\
\hline UFC & 10 & 9 & 8 & 7 & 11 & 9 \\
\hline UPM & 11 & 5 & 4 & 10 & - & 5 \\
\hline UNISINOS & 12 & 16 & 17 & 12 & 5 & - \\
\hline UFBA & 13 & 6 & 5 & 4 & 4 & 1 \\
\hline UFPE & 14 & 4 & 15 & 2 & 9 & - \\
\hline UFSC & 15 & 10 & 13 & 11 & 10 & - \\
\hline UERJ & 16 & 17 & 14 & 8 & - & 6 \\
\hline UFPR & 17 & 13 & 16 & 5 & - & 8 \\
\hline UNIFECAP & 18 & - & - & - & - \\
\hline UFAM & 18 & & & & & \\
\hline & 14 & & \\
\hline
\end{tabular}

Tabla 6. Ranking de PPGCC por área temática

Fuente: elaboración propia

Convenciones: R: ranking. CF: contabilidad financiera.

OUT: otras áreas relacionadas con contabilidad. CG: contabilidad gerencial. EPC: educación e investigación en contabilidad. AUD: auditoría. TB: tributación

La lectura de la tabla 7 permite afirmar que los programas FUCAPE, PUC/SP, UFBA, UFC, UFMG, UFPE, UFPR, UFRJ, UnB/UFPB/ UFRN, UNISINOS, UPM, USP y USP-RP concentran gran parte de la producción científica docente basada en la metodología empírica base de datos. 


\begin{tabular}{lcrrrr}
\hline Programas & EBD & EEC & EL & EPE & T \\
\hline FUCAPE & 75 & 3 & 2 & 2 & 17 \\
\hline FURB & 24 & 21 & 13 & 7 & 35 \\
\hline PUC-SP & 50 & 5 & 6 & 0 & 39 \\
\hline UERJ & 13 & 24 & 18 & 0 & 44 \\
\hline UFAM & 33 & 67 & 0 & 0 & 0 \\
\hline UFBA & 43 & 4 & 8 & 9 & 36 \\
\hline UFC & 45 & 22 & 2 & 0 & 31 \\
\hline UFMG & 53 & 19 & 2 & 1 & 25 \\
\hline UFPE & 44 & 15 & 15 & 5 & 22 \\
\hline UFPR & 48 & 16 & 10 & 3 & 23 \\
\hline UFRJ & 42 & 25 & 8 & 0 & 25 \\
\hline UFSC & 30 & 15 & 3 & 18 & 35 \\
\hline UNB/UFPB/ & 54 & 10 & 9 & 3 & 24 \\
\hline UFRN & 28 & 8 & 25 & 4 & 36 \\
\hline UNIFECAP & 65 & 21 & 2 & 3 & 9 \\
\hline UNISINOS & 46 & 2 & 9 & 15 & 28 \\
\hline UPM & 59 & 2 & 4 & 2 & 32 \\
\hline USP & 49 & 12 & 3 & 3 & 32 \\
\hline USP-RP & & & &
\end{tabular}

Tabla 7. Análisis descriptivo de los artículos por metodología (\%)

Fuente: elaboración propia

Convenciones: EBD: investigación empírica base de datos. EEC: investigación empírica estudio de caso. EL: investigación empírica levantamiento. EPE: empírica investigación experimental. T: investigación teórica

La tabla 8 refleja que los programas FUCAPE, FURB, PUC/SP, UFBA, UFC, UFMG, UFRJ, UnB/UFPB/UFRN, UNISINOS, UPM, USP y USP-RP concentran gran parte de la producción docente referente al área temática de contabilidad financiera. Los programas UFPE, UFPR, UFSC, UERJ y UNIFECAP concentran gran parte de la producción científica docente relacionada con el área temática de educación e investigación y otras áreas vinculadas, directa o indirectamente, con contabilidad. Por úl- timo, se puede afirmar que el programa de la UFAM se centra en investigación en otras áreas de contabilidad mientras que el programa de la FURB cuenta con especialistas en contabilidad financiera y gerencial.

\begin{tabular}{lcccccc}
\hline Programas & AUD & CF & CG & EPC & OUT & TB \\
\hline FUCAPE & 0 & 71 & 7 & 1 & 21 & 0 \\
\hline FURB & 7 & 32 & 31 & 13 & 17 & 0 \\
\hline PUC-SP & 7 & 60 & 14 & 3 & 16 & 0 \\
\hline UERJ & 6 & 17 & 20 & 10 & 47 & 0 \\
\hline UFAM & 0 & 33 & 0 & 0 & 67 & 0 \\
\hline UFBA & 12 & 50 & 11 & 10 & 18 & 0 \\
\hline UFC & 5 & 53 & 15 & 0 & 27 & 0 \\
\hline UFMG & 3 & 51 & 23 & 1 & 19 & 2 \\
\hline UFPE & 6 & 19 & 24 & 16 & 26 & 9 \\
\hline UFPR & 0 & 17 & 25 & 34 & 16 & 8 \\
\hline UFRJ & 0 & 52 & 22 & 3 & 21 & 2 \\
\hline UFSC & 4 & 18 & 10 & 37 & 32 & 0 \\
\hline UNB/UFPB/ & 4 & 46 & 17 & 14 & 15 & 4 \\
\hline UFRN & 0 & 11 & 16 & 33 & 36 & 4 \\
\hline UNIFECAP & 0 & 33 & 26 & 9 & 29 & 3 \\
\hline UNISINOS & 2 & 36 & 19 & 16 & 25 & 2 \\
\hline UPM & 54 & 10 & 20 & 12 & 2 \\
\hline USP & 57 & 10 & 8 & 25 & 0 \\
\hline USP-RP & 0 & & & & & \\
\hline & 0 & & & & \\
\hline
\end{tabular}

Tabla 8. Análisis descriptivo de los artículos por área temática (continua) (\%)

Fuente: elaboración propia

Convenciones: CF: contabilidad financiera. OUT: otras áreas relacionadas con contabilidad. CG: contabilidad gerencial. EPC: educación e investigación en contabilidad. AUD: auditoría. TB: tributación

La tabla 9 compara los dos abordajes del estudio y muestra claramente que las investigaciones en contabilidad financiera, otros temas y tributación se fundamentan en la metodología empírica base de datos. Los estudios en audi- 
toría, por su parte, se basan en la metodología empírica levantamiento. En cuanto a contabilidad gerencial, esta se concentra en tres metodologías: empírica base de datos, empírica estudio de caso e investigación teórica. Por último, en el área de educación e investigación en contabilidad, por lo general se recurre a la metodología teórica.

\begin{tabular}{lcrrrr}
\hline $\begin{array}{c}\text { Área } \\
\text { temática }\end{array}$ & EBD & EEC & EL & EPE & T \\
\hline AUD & 15 & 7 & 37 & 8 & 32 \\
\hline CF & 71 & 5 & 1 & 1 & 21 \\
\hline CG & 31 & 29 & 4 & 2 & 34 \\
\hline EPC & 23 & 1 & 19 & 17 & 40 \\
\hline OTROS & 38 & 16 & 8 & 2 & 35 \\
\hline TB & 73 & 17 & 0 & 1 & 9 \\
\hline
\end{tabular}

Tabla 9. Análisis descriptivo de artículos por área temática y metodología (\%)

Fuente: elaboración propia

Convenciones: CF: contabilidad financiera. OUT: otras áreas relacionadas con contabilidad. CG: contabilidad gerencial. EPC: educación e investigación en contabilidad. AUD: auditoría. TB: tributación. EBD: investigación empírica base de datos. EEC: investigación empírica estudio de caso. EL: investigación empírica levantamiento. EPE: empírica investigación experimental. T: investigación teórica

\section{Consideraciones finales}

La particularidad de este estudio es la utilización del método de ranking, propuesto por Joshua G. Coyne, Scott L. Summers, Brady Williams y David A. Wood (2009) para ayudar a resolver el problema de la investigación, pues hace más comprensible la interpretación de los resultados. El ranking evidencia de modo general la metodología empleada y el área temática de cada artículo de la producción docente, según la ventana temporal correspondiente. El número de artículos publicados se incrementó $450 \%$ en 2009 respecto a 2000, el 60\% de los artículos se publica con más de dos autores y el 1\% por sextetos. Hay una concentración del $44 \%$ de artículos en revistas que están clasificadas como B1 Qualis CAPES ${ }^{3}$.

La información general de la base de datos revela que la primera fase, que consistió en la catalogación de 27 revistas, generó 6.219 índices en la base de datos. Esos índices representan un total de 54.300 datos, que constituyen la población de la investigación. Para la construcción de los rankings, se seleccionó una muestra de 1.175 índices, para un total de 24.700 datos.

El ranking general de los PPGCC deja claro que el programa de USP es el más destacado. Los datos del estudio son compatibles con investigaciones realizadas anteriormente y con el resultado de la Evaluación Trienal de la CAPES. Estos datos indican que los programas de UFBA, UFPR y UFAM ocupan las últimas posiciones del ranking general.

Los PPGCC que más se destacan, en general, son los de la USP, FURB, FUCAPE, UFMG y USP-RP. Las principales características de estos programas son el considerable número de docentes de tiempo completo, la concentración de más del $80 \%$ de la producción docente publicada en revistas, el elevado número de

3 El Qualis constituye un sistema de evaluación periódica, mantenido por la Coordinación de Perfeccionamiento de Personal de Enseñanza Superior (CAPES), Brasil. CAPES lista y clasifica las revistas utilizadas para la difusión de la producción intelectual de los programas de estudios de postgrado en el ámbito de la circulación (locales, nacionales o internacionales) y de calidad (A, B, C), por área de evaluación. Extractos, en orden de calidad, son: A1, A2, B1, B2, B3, B4, B5 y C. 
investigaciones empíricas positivistas que emplean modelos cuantitativos, investigaciones enfocadas en el área financiera, la autoría compartida de artículos científicos en dúos y cuartetos principalmente. También se caracterizan por estar ubicados físicamente en las regiones geográficas del sudeste y sur de Brasil y por su desempeño creciente durante los 10 años que fueron objeto de este estudio.

El análisis descriptivo basado en la distribución de la frecuencia relativa indica que la metodología más empleada por los PPGCC es la empírica base de datos, mientras que el área temática más explorada es contabilidad financiera. Para estudios en contabilidad financiera, tributación y otros temas relacionados con contabilidad se utiliza la metodología empírica base de datos como procedimiento.

El estudio se limitó a revistas brasileñas disponibles electrónicamente, publicadas en un lapso de 10 años. Así, revistas que no cuentan con versión digital fueron excluidas. Los autores y coautores no fueron seleccionados; cada publicación representa un puntaje específico para cada investigador. La información acerca de la vinculación de los docentes fue verificada por medio del currículo Lattes, cuya actualización es responsabilidad de cada profesor.

Una reflexión necesaria es que Brasil, en 2009 , contaba con cuatro programas de posgrado que ofrecían formación de doctorado. La USP, según los datos de CAPES, comenzó a trabajar en 1978 para la instauración del segundo programa. Esto se logró en 2007, después de 29 años. El consorcio UnB/UFPB/UFRN fue instaurado y se constituyó en el segundo programa de doctorado en Brasil. En 2008, FURB estable- ció el tercero y, por último, FUCAPE instauró el cuarto en 2009. Una crítica válida, en este caso, sería que de los 18 PPGCC en vigencia, solamente 4 ofrecen doctorado en ciencias contables, lo cual es todavía muy poco en comparación con otras áreas del conocimiento.

En ese orden de ideas, tanto el problema como los objetivos de la investigación fueron alcanzados. Por último, como complemento de la investigación, cabe formular las siguientes recomendaciones para estudios futuros: (a) reproducir y expandir la investigación incluyendo revistas internacionales y aquellas disponibles únicamente en versión impresa; (b) realizar un estudio similar incluyendo tesis de maestría y de doctorado.

\section{Referencias}

\section{Beuren, Ilse Maria (2009). Como elaborar} trabalhos monográficos em contabilidade. Teoria e prática. 3 ed. São Paulo: Atlas. Borba, José Alonso \& Murcia, Fernando Dal-Ri (2006). Oportunidades para pesquisa e publicação em contabilidade: um estudo preliminar sobre as Revistas Acadêmicas de Língua Inglesa do Portal de Periódicos da CAPES. BBR, Brazilian Business Review, 3 (1), 88-103, enero/junio. Disponible en: http://www.redalyc.org/ pdf/1230/123016269007.pdf

Cardoso, Ricardo Lopes; Neto, Octávio Ribeiro de Mendonça; Riccio, Edson Luiz \& Sakata, Marici Cristine Gramacho (2005). Pesquisa científica em contabilidade entre 1990 e 2003. Revista de Administração de Empresas, 45 (2), 34-45. Disponible en: http://www. scielo.br/pdf/rae/v45n2/v45n2a04.pdf 
Coyne, Joshua G.; Summers, Scott L.; Williams, Brady \& Wood, David A. (2009). Accounting Program Research Rankings by Topical Area and Methodology. Brigham Young University. Disponible en https:// tippie.uiowa.edu/accounting/accounting rankings.pdf

Cruz, Carla \& Ribeiro, Uirá (2004). Metodologia científica: teoria e prática. 2 ed. Rio de Janeiro: Axcel Books.

Espejo, Márcia Maria dos Santos Bortolocci; Cruz, Ana Paula Capuano da; Lourenço, Rosenery Loureiro; Antonovz, Tatiane \& Almeida, Lauro Brito de (2009). Estado da arte da pesquisa contábil: um estudo bibliométrico de periódicos nacional e internacionalmente veiculados entre 2003 e 2007. Revista de Informação Contábil, 3 (3), 94-116. Disponible en: http://www.revista. ufpe.br/ricontabeis/index.php/contabeis/ article/viewFile/166/152

Francelin, Marivalde Moacir (2004). Ciência, senso comum e revoluções científicas: ressonâncias e paradoxos. Ciência $d a$ Informação, 33 (3), 26-34. Disponible en: http://www.scielo.br/pdf/ci/v33n3/ a04v33n3

Frezatti, Fábio (2000). Análise dos traços de tendência de uma amostra das revistas científicas da área de contabilidade publicadas na língua inglesa. Caderno de Estudos, 13 (24), 50-78. Disponible en: http://www.eac.fea.usp.br/cadernos/ completos/cad24/Revista_24_Art\%204.pdf

Fülbier, Rolf Uwe \& Sellhorn, Thorsten (2009). Approaches to Accounting Research Evidence from EAA Annual Congresses.
Social Science Research Network. Working paper series. Disponible en: http://papers. ssrn.com/sol3/papers.cfm?abstract_ $\mathrm{id}=985119 \&$

Gil, Antônio Carlos (2010). Como elaborar projetos de pesquisa. 5 ed. São Paulo: Atlas. Kuhn, Thomas S. (1979). Lógica da descoberta ou psicologia da pesquisa? En Imre Lakatos \& Alan Musgrave (org.). A crítica e o desenvolvimento do conhecimento. Octávio Mendes Cajado (trad.). São Paulo: Cultrix.

Lakatos, Eva Maria \& Marconi, Marina de Andrade (2010). Fundamentos de metodologia científica. 7 ed. São Paulo: Atlas. Disponible en: http://docente.ifrn.edu.br/ olivianeta/disciplinas/copy_of_historia-i/ historia-ii/china-e-india

Lakatos, Imre (1979). O falseamento e a metodologia dos programas de pesquisa científica. En Imre Lakatos \& Alan Musgrave (org.). A crítica e o desenvolvimento do conhecimento. Octávio Mendes Cajado (trad.). São Paulo: Cultrix. Leite-Filho, Geraldo Alemandro (2004). A relação orientador-orientando e suas influências no processo de elaboração de teses e dissertações dos programas de pós-graduação em contabilidade da cidade de São Paulo. São Paulo. Disertación. (Maestría en Ciencias Contables) - Programa de Posgrado en Ciencias Contables - Faculdade de Economia, Administração e Contabilidade da Universidades de São Paulo, FEA/USP. Disponible en: http://www.teses.usp.br/ teses/disponiveis/12/12136/tde-29012005165626/pt-br.php 
Leite-Filho, Geraldo Alemandro (20062008). Padrões de produtividade de autores em periódicos e congressos na área de contabilidade no Brasil: um estudo bibliométrico. En Congresso USP de Controladoria e Contabilidade, 6. Publicado en Revista de Administração Contemporânea, RAC, 12 (2), 533-554. Disponible en: http:// www.scielo.br/pdf/rac/v12n2/a11v12n2

Lyrio, Maurício Vasconcelos Leão; Borba, José Alonso \& Costa, Jeane Maria da (2007). Controle gerencial: delineamento do perfil metodológico de uma amostragem de publicações acadêmicas nas áreas de administração e contabilidade de 2000 a 2004. Base - Revista de Administação e Contabilidade da Unisinos, 4 (2), 126-136. Disponible en: http://www.spell.org.br/ documentos/download/20415

Machado-da-Silva, Clóvis L.; Fonseca, Valéria Silva da \& Crubellate, João Marcelo (2005). Estrutura, Agência e interpretação: elementos para a uma abordagem recursiva do processo de institucionalização. Revista de Administração Contemporânea, RAC, edición especial, 09-39. Disponible en: http:// www.scielo.br/scielo.php?pid=S1415$65552005000500002 \&$ script $=$ sci_arttext

Marinho, Iracema (2007). A comunicação científica e o modelo de comunicação organizacional: análise quantitativa de produtividade dos programas de pósgraduação em ciência da informação por meio do currículo Lattes. Brasília. Disertación (Maestría en Ciencias de la Información) - Universidade de Brasília.
Disponible en: http://repositorio.unb. br/bitstream/10482/3443/1/2007_ IracemaMarinho.pdf

Martins, Gilberto de Andrade \& Theóphilo, Carlos Renato (2009). Metodologia da investigação científica para ciências sociais aplicadas. 2. ed. São Paulo: Atlas.

Mendonça-Neto, Octávio Ribeiro de; Riccio, Edson Luiz \& Sakata, Marici Cristine Gramacho (2008). Dez anos de pesquisa contábil no Brasil: análise dos trabalhos apresentados nos ENANPADS de 1996 a 2005. Revista de Administração de Empresas, 49 (1), 62-73. Disponible en: http://www. scielo.br/pdf/rae/v49n1/v49n1a08.pdf Nogueira, Eloy Eros da Silva (2007). O processo de estruturação dos cursos de ensino superior da administração em Curitiba. São Paulo. Tesis (Doctorado en Administración) Escola de Administração de Empresas, Fundação Getúlio Vargas. Disponible en: http://bibliotecadigital.fgv.br/dspace/ handle/10438/4541

Oliveira, Marcelle Colares (2002). Análise dos Periódicos Brasileiro de Contabilidade. Revista Contabilidade \& Finanças - USP, São Paulo, 13 (29), 68-86. Disponible en: http:// www.scielo.br/scielo.php?script $=$ sci_arttext \&pid=S1519-70772002000200005

Peci, Alketa (2005-2006). A nova teoria institucional em estudos organizacionais: uma abordagem crítica. En Encontro da Associação Nacional de Pós-Graduação e Pesquisa em Administração, 29. Brasília. Anais. Rio de Janeiro: Associação Nacional dos Programas de Pós-Graduação em Administração, 2005. Publicado en 
RANKING DE LOS PROGRAMAS DE POSGRADO EN CONTABILIDAD EN BRASIL / A. COSTA, P. LUSTOSA / 57

Cadernos Ebape, 4 (1), 1-12. Disponible en: http://www.scielo.br/pdf/cebape/v4n1/ v4n1a06

Popper, Karl R. (1979). A ciência normal e seus perigos. En Imre Lakatos \& Alan Musgrave (org.). A crítica e o desenvolvimento do conhecimento. Octávio Mendes Cajado (trad.). São Paulo: Cultrix.

Riccio, Edson Luiz; Carastan, Jacira Tudora \& Sakata, Marici Cristine Gramacho (1999). Accounting Research at Brazilian Universities: 1962-1999. Caderno de Estudos, Fundação Instituto de Pesquisas Contábeis, Atuariais e Financeiras, 11 (22), 35-44. Disponible en: http:// www.scielo.br/scielo.php?pid=S141392511999000300004\&script=sci_arttext

Russell, Bertrand (1967). O impacto da ciência na sociedade. George Allen \& Unwin Ltd. (trad.). Rio de Janeiro: Zahar Editores.

Sem Surpresa, Harvard lidera ranking de universidades. Estadão, Educação, São Paulo, 16 sep. 2010. Disponible en: http:// www.estadao.com.br/noticias/vidae,semsurpresa-harvard-lidera-ranking-deuniversidades,610820,0.htm

Silveira, Fernando Lang da (1996). A metodologia dos Programas de Pesquisa: a epistemologia de Imre Lakatos. Revista Caderno Cararinense de Ensino de Física, Florianópolis, 13 (3), 219-230. Disponible en: https://www.lume.ufrgs.br/bitstream/ handle/10183/85015/000190613. pdf?sequence $=1$

Souza, Flávia Cruz de; Rover, Suliani; Gallon, Alessandra Vasconcelos \& Ensslin, Sandra Rolim (2008). Análise das IES da área de ciências contábeis e de seus pesquisadores por meio de sua produção científica. Revista Contabilidade Vista e Revista, 19 (3), 15-38. Disponible en: http://www.spell.org.br/ documentos/download/8090

Theóphilo, Carlos Renato \& Iudícibus, Sérgio de (2005). Uma análise críticoepistemológica da produção científica em contabilidade no Brasil. En Encontro da Associação Nacional de Pós-Graduação e Pesquisa em Administração, 29. Brasília. Anais, Brasília. Publicado en Revista UnB Contábil - UnB, Brasília, 8 (2), 147-175. Disponible en: http://www.cgg-amg.unb.br/ index.php/contabil/article/view/164/pdf_87

Universidade de São Paulo, 10 Congresso USP de Contabilidade e Controladoria (2010). Áreas temáticas. Disponible en: http://www. congressousp.fipecafi.org/

Walter, Silvana Anita; Cruz, Ana Paula Capuano da; Espejo, Márcia Maria dos Santos Bortolocci \& Gassner, Flavia Pozzera (2009). Uma análise da evolução do campo de ensino e pesquisa em contabilidade sob a perspectiva de redes. Revista Universo Contábil, 5 (4), 76-93. Disponible en: http://www.spell.org.br/documentos/ download/6232

- Fecha de recepción: 2 de julio de 2013

- Fecha de aceptación: 28 de febrero de 2014

- Disponible en línea: 01 de julio de 2014 


\section{Para citar este artículo}

Costa, Abimael de Jesus Barros \& Lustosa, Paulo Roberto Barbosa (2014). Rankings de los programas de posgrado en contabilidad: análisis de producción docente según publicación en revistas brasileñas (2000-2009). Cuadernos de Contabilidad, 15 (38), 549-573.

doi: 10.11144/Javeriana.cc15-38.rppc

\section{Anexo A}

\section{Definición de las estrategias de} investigación

Teórica: investigación bibliográfica que analiza los trabajos anteriores, revestidos de importância. La investigación teórica recurre a fuentes primarias y fuentes secundarias y está conformada por trabajos no originales. Investigación documental que utiliza material que aún no ha recibido tratamiento analítico (Cruz \& Ribeiro, 2004, p. 19; Lakatos \& Marconi, 2010, p. 159; Fülbier \& Sellhorn, 2009, p. 24).

Empírica base de datos: agrupa investigaciones en que se emplean, principalmente, técnicas estadísticas sofisticadas (Fülbier \& Sellhorn, 2009, p. 24).

Empírica levantamiento: agrupa estudios descriptivos, cuya principal finalidad es la descripción o análisis de las características, hechos y fenómenos, la evaluación de programas, o el aislamiento de variables principales o clave (Lakatos \& Marconi, 2010, pp. 187-188; Fülbier \& Sellhorn, 2009, p. 24).

Empírica estudio de caso: incluye estudios in situ en entidades públicas y privadas. El término estudio de caso implica en general una investigación dedicada a una única unidad de análisis, que puede ser un departamento, empresa, industria o incluso otros países (Fülbier \& Sellhorn, 2009, p. 24).

Empírica investigación experimental: agrupa investigaciones experimentales que son investigaciones empíricas cuyo objetivo principal es la verificación de hipótesis acerca de relaciones causa-efecto. Este tipo de investigación se caracteriza por grupos de control, selección de muestra por técnicas probabilísticas y manipulación de variables independientes (Lakatos \& Marconi, 2010, p. 189; Fülbier \& Sellhorn, 2009, p. 24).

\section{Anexo B \\ Definición de áreas temáticas}

Auditoría: investigaciones científicas que abordan asuntos relacionados con temas generales sobre auditoría, auditoría interna y externa, auditoría independiente (Coyne, Summers, Williams \& Wood, 2010; Fülbier \& Sellhorn, 2009; Oliveira, 2002; $10^{\circ}$ Congresso USP de Contabilidade e Controladoria, 2010).

Contabilidad gerencial: investigaciones científicas que impliquen el desarrollo de instrumentos de apoyo al proceso de planeación y control gerencial de las entidades (Coyne, Summers, Williams \& Wood, 2010; Fülbier \& Sellhorn, 2009; Oliveira, 2002; $10^{\circ}$ Congresso USP de Contabilidade e Controladoria, 2010).

Contabilidad financiera: investigaciones científicas que abordan temas como contabilidad comercial, contabilidad de sociedades, contabilidad internacional, comparación entre diferentes normas, prácticas y principios que rigen la contabilidad en diversos países del mundo, variación en el precio de acciones, in- 
fluencia de las demostraciones contables en el mercado de capitales, mercado financiero y mercado de crédito (Coyne, Summers, Williams \& Wood, 2010; Fülbier \& Sellhorn, 2009; Oliveira, 2002; 10 Congresso USP de Contabilidade e Controladoria, 2010);

Tributación: investigaciones científicas que abordan temas como contabilidad tributaria (C; 10 Congresso USP de Contabilidade e Controladoria, 2010);

Educación e investigación en contabilidad: investigaciones científicas que abordan estudios relacionados con educación contable, posgrado, técnicas e instrumentos que buscan el perfeccionamiento y mejoramiento de todo el proceso de enseñanza-aprendizaje y con la elaboración de trabajos científicos en todas las áreas del campo de conocimiento de ciencia contable (Coyne,
Summers, Williams \& Wood, 2010; Fülbier \& Sellhorn, 2009; Oliveira, 2002; 10 Congresso USP de Contabilidade e Controladoria, 2010).

Otros: temas relacionados con contabilidad gubernamental, presupuesto público, finanzas públicas, contabilidad social y ambiental, contabilidad aplicada al tercer sector, pericia contable, cálculos actuariales, sistemas de información contable, aspectos contables específicos en entidades inmobiliarias, cooperativas, agropecuarias, ejercicio de la profesión contable, ética profesional, metodología de la investigación en contabilidad, métodos cuantitativos aplicados a contabilidad, psicología, derecho y clima organizacional (Coyne, Summers, Williams \& Wood, 2010; Fülbier \& Sellhorn, 2009; Oliveira, 2002; 10 Congresso USP de Contabilidade e Controladoria, 2010). 
